Received: 25 June 2013, Accepted: 10 December 2013

Edited by: R. Dickman

Reviewed by: F. Reis, Instituto de Física, Univ. Fed. Fluminense, Brazil.

Licence: Creative Commons Attribution 3.0

DOI: http://dx.doi.org/10.4279/PIP.050010

www.papersinphysics.org

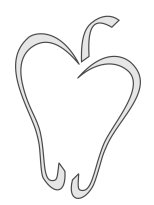

ISSN 1852-4249

\title{
Invited review: KPZ. Recent developments via a variational formulation
}

\author{
Horacio S. Wio, ${ }^{1 *}$ Roberto R. Deza,${ }^{2 \dagger}$ Carlos Escudero, ${ }^{3 \ddagger}$ Jorge A. Revelli ${ }^{4 \S}$
}

\begin{abstract}
Recently, a variational approach has been introduced for the paradigmatic Kardar-ParisiZhang (KPZ) equation. Here we review that approach, together with the functional Taylor expansion that the KPZ nonequilibrium potential (NEP) admits. Such expansion becomes naturally truncated at third order, giving rise to a nonlinear stochastic partial differential equation to be regarded as a gradient-flow counterpart to the KPZ equation. A dynamic renormalization group analysis at one-loop order of this new mesoscopic model yields the KPZ scaling relation $\alpha+z=2$, as a consequence of the exact cancelation of the different contributions to vertex renormalization. This result is quite remarkable, considering the lower degree of symmetry of this equation, which is in particular not Galilean invariant. In addition, this scheme is exploited to inquire about the dynamical behavior of the KPZ equation through a path-integral approach. Each of these aspects offers novel points of view and sheds light on particular aspects of the dynamics of the KPZ equation.
\end{abstract}

\section{Introduction}

Although readers whose careers span mostly on the 21th century might not care about this, back in the sixties (when transistors and lasers had already been invented) equilibrium critical phenomena were still a puzzle. In fact, although a sense of "universality" had been gained in 1950 through a field theory based on the innovative concept of order parameter $[1,2]$, its predicted critical exponents were

\footnotetext{
*E-mail: wio@ifca.unican.es

†E-mail: deza@mdp.edu.ar

${ }^{\ddagger}$ E-mail: cel@icmat.es

$\S$ E-mail: revelli@famaf.unc.edu.ar

1 IFCA (UC-CSIC), Avda. de los Castros s/n, E-39005 Santander, Spain.

2 IFIMAR (UNMdP-CONICET), Funes 3350, 7600 Mar del Plata, Argentina.

3 Depto. Matemáticas \& ICMAT (CSIC-UAM-UC3MUCM), Cantoblanco, E-28049 Madrid, Spain.

4 FaMAF-IFEG (CONICET-UNC), 5000 Córdoba, Argentina.
}

almost as a rule wrong. It was not until the seventies that a far more sophisticated field-theory approach [3] brought order home: equilibrium universality classes are determined solely by the dimensionalities of the order parameter and the ambient space. Since then, one of Statistical Physics' "holy grials" has been to conquer a similar achievement for non-equilibrium critical phenomena [4]. In such a (still unaccomplished) enterprise, a valuable field-theoretical tool has been in the last quarter of century the Kardar-Parisi-Zhang (KPZ) equation $[5-7]$.

The KPZ equation [5-7] has become a paradigm for the description of a vast class of nonequilibrium phenomena by means of stochastic fields. The field $h(x, t)$, whose evolution is governed by this stochastic nonlinear partial differential equation, describes the height of a fluctuating interface in the context of surface-growth processes in which it was originally formulated. From a theoretical point of view, the KPZ equation has many interesting properties, for instance, its close relationship with the Burgers equation [8] or with a dif- 
Papers in Physics, vol. 5, ART. 050010 (2013) / H. S. Wio et al.

fusion equation with multiplicative noise, whose field $\phi(x, t)$ can be interpreted as the restricted partition function of the directed polymer problem [9]. Many of the efforts put in investigating the behavior of its solutions were focused on obtaining the scaling laws and critical exponents in one or more spatial dimensions [11-17]. However, other questions of great interest are the development of suitable algorithms for its numerical integration $[18,19]$, the construction of particular solutions [20-23], the crossover behavior between different regimes [10,24-26], as well as related ageing and pinning phenomena [27-29].

Among all the classical theoretical developments concerning this equation $[6,7]$, two have recently drawn our attention. One was the scaling relation $\alpha+z=2$, which is expected to be exact for the $\mathrm{KPZ}$ equation in any dimension. The exactness of this relation has been traditionally attributed to the Galilean invariance of the KPZ equation. Nevertheless, the assumed central role of this symmetry has been challenged in this as well as in other nonequilibrium models from both a theoretical [30-33] and a numerical [34-36] point of view. The second one is the generally accepted lack of existence of a suitable functional allowing to formulate the KPZ equation as a gradient flow. In fact, a variational approach to the closely related SunGuo-Grant [37] and Villain-Lai-Das Sarma [38, 39] equations was developed in $[40,41]$ by means of a geometric construction. In [46], a Lyapunov functional (with an explicit density) was found for the deterministic KPZ equation. Also, a nonequilibrium potential (NEP), a functional that allows the formal writing of the KPZ equation as a (stochastically forced) exact gradient flow, was introduced.

In this work we shortly review the consistency constraints imposed by the nonequilibriumpotential structure on discrete representations of the KPZ equation and show that they lead to explicit breakdown of Galilean invariance, despite the fact that the obtained numerical results are still those of the KPZ universality class. A Taylor expansion of the previously introduced NEP has (in terms of fluctuations) an explicit density and a thought-provoking structure [47], and leads to an equation of motion (for fluctuations, in the continuum) with exact gradient-flow structure, but different from the KPZ one. This equation has a lower degree of symmetry: it is neither Galilean invariant nor even translation invariant. Its scaling properties are studied by means of a dynamic renormalization group (DRG) analysis, and its critical exponents fulfill at one-loop order the same scaling relation $\alpha+z=2$ as those of the KPZ equation, despite the aforementioned lack of Galilean invariance. The concern with stability leads us to suggest the introduction of an equation related to the Kuramoto-Sivashinsky one, also with exact gradient-flow structure. We close this article exposing some novel developments based on a pathintegral-like approach.

\section{Brief review of the nonequilib- rium potential scheme}

Loosely speaking, the notion of NEP is an extension to nonequilibrium situations of that of equilibrium thermodynamic potential. In order to introduce it, we consider a general system of nonlinear stochastic equations (admitting the possibility of multiplicative noises)

$$
\dot{q}^{\nu}=K^{\nu}(q)+g_{i}^{\nu}(q) \xi_{i}(t), \quad \nu=1, \ldots, n ;
$$

where repeated indices are summed over. Equation (1) is stated in the sense of Itô. The $\left\{\xi_{i}(t)\right\}, i=$ $1, \ldots, m \leq n$ are mutually independent sources of Gaussian white noise with typical strength $\gamma$.

The Fokker-Planck equation corresponding to Eq. (1) takes the form

$$
\frac{\partial P}{\partial t}=-\frac{\partial}{\partial q^{\nu}} K^{\nu}(q) P+\frac{\gamma}{2} \frac{\partial^{2}}{\partial q^{\nu} \partial q^{\mu}} Q^{\nu \mu}(q) P
$$

where $P(q, t ; \gamma)$ is the probability density of observing $q=\left(q_{1}, \ldots, q_{n}\right)$ at time $t$ for noise intensity $\gamma$, and $Q^{\nu \mu}(q)=g_{i}^{\nu}(q) g_{i}^{\mu}(q)$ is the matrix of transport coefficients of the system, which is symmetric and non-negative. In the long time limit $(t \rightarrow \infty)$, the solution of Eq. (2) tends to the stationary distribution $P_{\text {st }}(q)$. According to [42-44], the $\operatorname{NEP} \Phi(q)$ associated to Eq. (2) is defined by

$$
\Phi(q)=-\lim _{\gamma \rightarrow 0} \gamma \ln P_{\mathrm{st}}(q, \gamma) .
$$

In other words,

$$
P_{\mathrm{st}}(q) d^{n} q=Z(q) \exp \left[-\frac{\Phi(q)}{\gamma}+\mathcal{O}(\gamma)\right] d \Omega_{q},
$$


PAPers in Physics, vol. 5, ART. 050010 (2013) / H. S. Wio et al.

where $\Phi(q)$ is the NEP of the system and the prefactor $Z(q)$ is defined as the limit

$$
\ln Z(q)=\lim _{\gamma \rightarrow 0}\left[\ln P_{\text {st }}(q, \gamma)+\frac{1}{\gamma} \Phi(q)\right] .
$$

Here $d \Omega_{q}=d^{n} q / \sqrt{G(q)}$ is the invariant volume element in the $q$-space and $G(q)$ is the determinant of the contravariant metric tensor (for the Euclidean metric it is $G=1)$. It was shown [42] that $\Phi(q)$ is the solution of a Hamilton-Jacobi-like equation (HJE)

$$
K^{\nu}(q) \frac{\partial \Phi}{\partial q^{\nu}}+\frac{1}{2} Q^{\nu \mu}(q) \frac{\partial \Phi}{\partial q^{\nu}} \frac{\partial \Phi}{\partial q^{\mu}}=0,
$$

and $Z(q)$ is the solution of a linear first-order partial differential equation depending on $\Phi(q)$ (not shown here).

Equation (3) and the normalization condition ensure that $\Phi$ is bounded from below. Furthermore, it follows that

$$
\frac{d \Phi(q)}{d t}=K^{\nu}(q) \frac{\partial \Phi(q)}{\partial q^{\nu}}=-\frac{1}{2} Q^{\nu \mu}(q) \frac{\partial \Phi}{\partial q^{\nu}} \frac{\partial \Phi}{\partial q^{\mu}} \leq 0,
$$

i.e., $\Phi$ is a Lyapunov functional for the dynamics of the system when fluctuations are neglected. Under the deterministic dynamics, $\dot{q}^{\nu}=K^{\nu}(q)$, $\Phi$ decreases monotonically and takes a minimum value on attractors. In particular, $\Phi$ must be constant on all extended attractors (such as limit cycles or strange attractors) [42].

An alternative way to look into this problem is due to Ao [45]. The interesting feature of this approach is that it resorts neither to $P_{s t}(q)$ nor to the small-noise limit, thus being applicable in principle to more general situations.

\section{Variational approach for KPZ}

The Kardar-Parisi-Zhang (KPZ) equation reads

$$
\frac{\partial h(x, t)}{\partial t}=\nu \nabla^{2} h(x, t)+\frac{\lambda}{2}[\nabla h(x, t)]^{2}+\xi(x, t),
$$

where $\xi(x, t)$ is a Gaussian white noise, of zero mean $(\langle\xi(x, t)\rangle=0)$ and correlation $\left\langle\xi(x, t) \xi\left(x^{\prime}, t^{\prime}\right)\right\rangle=2 \gamma \delta\left(x-x^{\prime}\right) \delta\left(t-t^{\prime}\right)$. As it is well known, this nonlinear differential equation describes the fluctuations of a growing interface with a surface tension given by $\nu ; \lambda$ is proportional to the average growth velocity and arises because the surface slope is paralleled transported in such a growth process.

\section{Lyapunov functional}

The deterministic KPZ equation-obtained by setting $\gamma=0$-is exactly solvable by means of the Hopf-Cole transformation $\left(\phi(x, t)=e^{\frac{\lambda}{2 \nu} h(x, t}\right)$, which maps the nonlinear KPZ equation onto the (deterministic) linear diffusion equation [5]

$$
\frac{\partial \phi(x, t)}{\partial t}=\nu \nabla^{2} \phi(x, t)
$$

Also, the multiplicative reaction-diffusion (RD) equation

$$
\frac{\partial \phi(x, t)}{\partial t}=\nu \nabla^{2} \phi(x, t)+\phi(x, t) \xi(x, t),
$$

which is associated to the directed polymer problem $[6,7,9]$ results, using the inverse transformation $\left(h(x, t)=\frac{2 \nu}{\lambda} \ln \phi(x, t)\right)$ to be mapped into the complete KPZ equation (4).

The deterministic part of Eq. (6) (i.e., Eq. (5)), can be written as

$$
\frac{\partial \phi(x, t)}{\partial t}=-\frac{\delta \mathcal{F}[\phi(x, t)]}{\delta \phi(x, t)}
$$

where $\mathcal{F}[\phi(x, t)]$ is the Lyapunov functional of the deterministic RD problem given by

$$
\mathcal{F}[\phi(x, t)]=\frac{\nu}{2} \int[\nabla \phi(x, t)]^{2} \mathrm{~d} x .
$$

Applying to this functional the above indicated inverse transformation we get [46]

$$
\mathcal{F}[h]=\frac{\lambda^{2}}{8 \nu} \int e^{\frac{\lambda}{\nu} h(x, t)}[\nabla h(x, t)]^{2} \mathrm{~d} x,
$$

that allows the KPZ equation to be written as

$$
\frac{\partial}{\partial t} h(x, t)=-\Gamma[h] \frac{\delta \mathcal{F}[h]}{\delta h(x, t)}+\xi(x, t) .
$$

One can check the Lyapunov property $\dot{\mathcal{F}}[h] \leq 0$, with the motility $\Gamma[h]$ given by

$$
\Gamma[h]=\left(\frac{2 \nu}{\lambda}\right)^{2} e^{-\frac{\lambda}{\nu} h(x, t)},
$$

and that its minimum is achieved by constant functions. Hence we have a Lyapunov functional for the deterministic KPZ equation that displays simple dynamics: the asymptotic stability of constant 
Papers in Physics, vol. 5, ART. 050010 (2013) / H. S. Wio et al.

solutions indicates an approach to constant profiles at long times, for arbitrary initial conditions. Despite this simplicity in the deterministic case, the stochastic situation is far from trivial and gives rise to self-affine fractal profiles. In particular, the existence of this Lyapunov functional provides no a priori intuition on the stochastic dynamics.

\section{The nonequilibrium potential}

An alternative functional was also proposed in [46]. By starting from the functional Fokker-Planck equation, we look for the stationary solution (in fact steady-state solution), and after some integration by parts, it is possible to arrive to another form of Lyapunov functional

$\Phi[h]=\frac{\nu}{2} \int \mathrm{d} x(\nabla h)^{2}-\frac{\lambda}{2} \int \mathrm{d} x \int_{h_{\mathrm{ref}}}^{h(x, t)} \mathrm{d} \psi(\nabla \psi)^{2}$.

It is somehow inspired in the analytical form of "model A", according to the classification of critical phenomena in [48]. Here, the interpretation of the integral in the 2 nd term on the rhs is $\int \mathrm{d} x \int_{h_{\mathrm{ref}}}^{h(x, t)} \mathrm{d} \psi=\sum_{j} \triangle x \int_{h_{\mathrm{ref}, \mathrm{j}}}^{h_{j}} d \psi_{j}$. According to this definition, the KPZ equation can be formally written as a stochastically forced gradient flow

$$
\frac{\partial}{\partial t} h(x, t)=-\frac{\delta \Phi[h]}{\delta h(x, t)}+\xi(x, t) .
$$

The functional so defined fulfills the Lyapunov condition $\dot{\Phi}[h]=-\left(\frac{\delta \Phi[h]}{\delta h(x, t)}\right)^{2} \leq 0$ as well, and could be identified as the nonequilibrium potential (NEP) for the $\mathrm{KPZ}$ case $[53,54]$.

We will not pursue here the development of rigorous result concerning the functional (10). Our present interest falls in the calculation of quantities of physical interest rather than in building a completely rigorous mathematical theory. It is worth remarking that, as indicated in [46] and above, such a form has a discrete definition. It is also interesting to point out that analogous functionals involving functional integrals which are not carried out explicitly were obtained for the problem of interface fluctuations in random media [49-52].

\section{NEP expansion}

We now proceed to formally Taylor expand the NEP defined in Eq. (10) around a given reference (or initial) state, denoted by $h_{0}$

$$
\begin{aligned}
\Phi[h]=\frac{\nu}{2} \int \mathrm{d} x(\nabla h)^{2} \\
\quad-\frac{\lambda}{2} \int \mathrm{d} x \int_{h_{0}}^{h(x, t)} \mathrm{d} \psi(\nabla \psi)^{2} \\
\approx \Phi\left[h_{0}\right]+\delta \Phi\left[h_{0}\right]+\frac{1}{2} \delta^{2} \Phi\left[h_{0}\right] \\
+\frac{1}{6} \delta^{3} \Phi\left[h_{0}\right]+\cdots .
\end{aligned}
$$

The successive terms in the expansion of $\Phi[h]$ are

$$
\begin{aligned}
\delta \Phi\left[h_{0}\right] & =-\int \mathrm{d} x\left[\nu \nabla^{2} h_{0}+\frac{\lambda}{2}\left(\nabla h_{0}\right)^{2}\right] \delta h, \\
\delta^{2} \Phi\left[h_{0}\right] & =-\int \mathrm{d} x \delta h\left(\nu \nabla^{2}+\lambda \nabla h_{0} \cdot \nabla\right) \delta h \\
\delta^{3} \Phi\left[h_{0}\right] & =-\lambda \int \mathrm{d} x \delta h(\nabla \delta h)^{2} .
\end{aligned}
$$

Clearly, for higher order $(n \geq 4)$ terms we have

$$
\delta^{n} \Phi\left[h_{0}\right] \equiv 0
$$

indicating that this formal expansion has a natural cut-off after the third order.

It is worth indicating that in this computationas in all the other computations within this workboundary terms vanish provided one of the following types of boundary conditions is assumed: homogeneous Dirichlet boundary conditions, homogeneous Neumann boundary conditions, periodic boundary conditions or an infinite space with the derivatives of $\delta h$ vanishing as they approach an infinite distance from the origin.

The reference state $h_{0}$ is arbitrary (i.e., any initial condition), but it is particularly useful to take it as one that makes $\delta \Phi\left[h_{0}\right]=0$, that is: a solution to the stationary counterpart of the deterministic KPZ equation. The complete set of solutions is $h_{0}=c$, where $c$ is an arbitrary constant (arbitrary up to the application of the boundary conditions, whenever this consideration applies), what physically corresponds to a flat interface. Hence we have $\left(\delta h=h-h_{0}\right)$

$$
\Phi[h]=\Phi\left[h_{0}\right]+\frac{1}{2} \delta^{2} \Phi\left[h_{0}, \delta h\right]+\frac{1}{6} \delta^{3} \Phi\left[h_{0}, \delta h\right] .
$$


PAPers in Physics, vol. 5, ART. 050010 (2013) / H. S. Wio et al.

\section{The equation for fluctuations}

From here we can define an effective NEP, which drives the dynamics of the fluctuations $\delta h$ and has an explicit density. Clearly, it corresponds to the last two terms in Eq. (15). To simplify the notation we adopt $u(x, t):=\delta h(x, t)$, and so the NEP reads

$$
\begin{aligned}
\mathcal{I}[u] & =\int \mathrm{d} x\left[\frac{\nu}{2}-\frac{\lambda}{6} u(x, t)\right](\nabla u)^{2} \\
& =-\int \mathrm{d} x u(x, t)\left[\nu \nabla^{2} u+\frac{\lambda}{6}(\nabla u)^{2}\right] .
\end{aligned}
$$

The deterministic equation for $u$ results

$$
\begin{aligned}
& \frac{\partial u}{\partial t}=-\frac{\delta \mathcal{I}[u]}{\delta u}, \\
& \frac{\partial u}{\partial t}=\left(\nu-\frac{\lambda u}{3}\right) \nabla^{2} u-\frac{\lambda}{6}(\nabla u)^{2} .
\end{aligned}
$$

Clearly, patterns like $u_{0}=$ constant are stationary solutions of Eq. (17): for all of them $\mathcal{I}[u]=0$, indicating that all such states have the same "energy". Finally, let us remark that although the formal Taylor expansion becomes naturally truncated at third order, the deterministic KPZ equation is not recovered. We call the stochastic version of this new equation "KPZW".

There is a remarkable difference between both equations (KPZ and Eq. (17)). It arises due to the fact that in the first case we have a fixed equation for $h$ and for any initial condition, while in the second case we have a fixed initial condition $(u=0)$ with a variable equation whose coefficients depend on $h_{o}$ ! (it is an equation for the departure from the given initial condition). The question of the relevance of this aspect to ageing problems (as discussed for instance in [29]) arises naturally. This point, worth to be analyzed, will be the subject of further work.

\section{Non-local kernel}

In previous works $[36,46]$ it was indicated that the following functional, including a nonlocal contribution,

$$
\begin{aligned}
\mathcal{F}[h]= & \int_{\Omega}\left\{\left(\frac{\lambda^{2}}{8 \nu}\right)(\nabla h)^{2}+e^{-\frac{\lambda}{2 \nu} h(\mathbf{x}, t)}\right. \\
& \left.\times \int_{\Omega} d \mathbf{x}^{\prime} G\left(\mathbf{x}, \mathbf{x}^{\prime}\right) e^{\frac{\lambda}{2 \nu} h\left(\mathbf{x}^{\prime}, t\right)}\right\} e^{\frac{\lambda}{\nu} h(\mathbf{x}, t)} d \mathbf{x}
\end{aligned}
$$

leads, after functional derivation, to a generalized KPZ equation

$$
\begin{aligned}
\partial_{t} h(\mathbf{x}, t)= & \nu \nabla^{2} h(\mathbf{x}, t)+\frac{\lambda}{2}[\nabla h(\mathbf{x}, t)]^{2} \\
& -e^{-\frac{\lambda}{2 \nu} h(\mathbf{x}, t)} \int_{\Omega} d \mathbf{x}^{\prime} G\left(\mathbf{x}, \mathbf{x}^{\prime}\right) e^{\frac{\lambda}{2 \nu} h\left(\mathbf{x}^{\prime}, t\right)} \\
& +\xi(\mathbf{x}, t) .
\end{aligned}
$$

It was also shown that if the nonlocal kernel has translational invariance $\left(G\left(\mathbf{x}, \mathbf{x}^{\prime}\right)=G\left(\mathbf{x}-\mathbf{x}^{\prime}\right)\right)$, and also, if it is of (very) "short" range, it can be expanded as

$$
G\left(\mathbf{x}-\mathbf{x}^{\prime}\right)=\sum_{n=0}^{\infty} A_{2 n} \delta^{(2 n)}\left(\mathbf{x}-\mathbf{x}^{\prime}\right),
$$

with $\delta^{(n)}\left(\mathbf{x}-\mathbf{x}^{\prime}\right)=\nabla_{\mathbf{x}^{\prime}}^{n} \delta\left(\mathbf{x}-\mathbf{x}^{\prime}\right)$, and where symmetry properties were taken into account. Exploiting this form of the kernel, and considering different approximation orders, it is possible to recover contributions having the same form as the ones arising in several previous works, where scaling properties, symmetry arguments, etc., have been used to discuss the possible contributions to a general form of the kinetic equation [55-57]. Such different contributions are tightly related to several of other previously studied equations, like the Sun-Guo-Grant equation [37], as well as others [55, 58].

We will not pursue this aspect here, but we will briefly refer again to it in a forthcoming section.

\section{Discretization issues, symmetry violation and all that}

In this section we will review aspects related to two main symmetries associated with the $1 \mathrm{D} \mathrm{KPZ}$ equation: Galilean invariance and the fluctuationdissipation relation. On the one hand, Galilean invariance has been traditionally linked to the exactness of the relation $\alpha+z=2$ among the critical exponents, in any spatial dimensionality (the roughness exponent $\alpha$, characterizing the surface morphology in the stationary regime, and the dynamic exponent $z$, indicating the correlation length scaling as $\left.\xi(t) \sim t^{1 / z}\right)$. However, this interpretation has been criticized in this and other nonequilibrium models $[31,32,59]$. On the other hand, the second symmetry essentially tells us that in $1 \mathrm{D}$, the nonlinear (KPZ) term is not operative at long times. 
PAPers in Physics, vol. 5, ART. 050010 (2013) / H. S. Wio et al.

Even when recognizing the interesting analytical properties of the KPZ equation, it is clear that investigating the behavior of its solutions requires the (stochastic) numerical integration of a discrete version. Such an approach has been used ,e.g., to obtain the critical exponents in one and more spatial dimensions [10-15,60]. Although a pseudo-spectral spatial discretization scheme has been recently introduced $[18,61]$, real-space discrete versions of Eq. (4) are still used for numerical simulations $[62,63]$. One reason is their relative ease of implementation and of interpretation in the case of nonhomogeneous substrates, for example a quenched impurity distribution [64].

\section{Consistency}

Here, we use the standard, nearest-neighbor discretization prescription as a benchmark to elucidate the constraints to be obeyed by any spatial discretization scheme, arising from the mapping between the KPZ and the diffusion equation (with multiplicative noise) through the Hopf-Cole transformation.

The standard spatially discrete version of Eq. (6) is

$$
\dot{\phi}_{j}=\frac{\nu}{a^{2}}\left(\phi_{j+1}-2 \phi_{j}+\phi_{j-1}\right)+\frac{\lambda \sqrt{\gamma}}{2 \nu} \phi_{j} \xi_{j},
$$

with $1 \leq j \leq N \equiv 0$, because of the assumed periodic b.c. (the implicit sum convention is not meant in any of the discrete expressions). Here $a$ is the lattice spacing. Then, using the discrete version of Hopf-Cole transformation $\phi_{j}(t)=\exp \left[\frac{\lambda}{2 \nu} h_{j}(t)\right]$, we get

$$
\dot{h}_{j}=\frac{2 \nu^{2}}{\lambda a^{2}}\left(\mathrm{e}^{\delta_{j}^{+} a}+\mathrm{e}^{\delta_{j}^{-} a}-2\right)+\sqrt{\gamma} \xi_{j},
$$

with $\delta_{j}^{ \pm} \equiv \frac{\lambda}{2 \nu a}\left(h_{j \pm 1}-h_{j}\right)$. By expanding the exponentials up to terms of order $a^{2}$, and collecting equal powers of $a$ (observe that the zero-order contribution vanishes) we retrieve

$$
\begin{aligned}
\dot{h}_{j}= & \frac{\nu}{a^{2}}\left(h_{j+1}-2 h_{j}+h_{j-1}\right) \\
& +\frac{\lambda}{4 a^{2}}\left[\left(h_{j+1}-h_{j}\right)^{2}+\left(h_{j}-h_{j-1}\right)^{2}\right] \\
& +\sqrt{\gamma} \xi_{j} .
\end{aligned}
$$

As we can see, the first and second terms on the r.h.s. of Eq. (23) are strictly related by virtue of the
Hopf-Cole transformation. In other words, the discrete form of the Laplacian in Eq. (21) constrains the discrete form of the nonlinear term in the transformed equation. Later we return, in another way, to the tight relation between the discretization of both terms. Known proposals [60] fail to comply with this natural requirement.

An important feature of the Hopf-Cole transformation is that it is local, i.e., it involves neither spatial nor temporal transformations. An effect of this feature is that the discrete form of the Laplacian is the same, regardless of whether it is applied to $\phi$ or $h$.

The aforementioned criterion dictates the following discrete form for $\mathcal{F}[\phi]$ (the one just before Eq. (8)), thus a Lyapunov function for any finite $N$

$$
\begin{aligned}
\mathcal{F}[\phi] & =\frac{\nu}{2} \sum_{j=1}^{N} a\left(\left(\partial_{x} \phi\right)^{2}\right)_{j} \\
& =\frac{\nu}{4 a} \sum_{j=1}^{N}\left[\left(\phi_{j+1}-\phi_{j}\right)^{2}+\left(\phi_{j}-\phi_{j-1}\right)^{2}\right]
\end{aligned}
$$

It is a trivial task to verify that the Laplacian is $\left(\partial_{x}^{2} \phi\right)_{j}=-a^{-1} \partial_{\phi_{j}} \mathcal{F}[\phi]$. Now, the obvious fact that this functional can also be written as $\mathcal{F}[\phi]=$ $\frac{\nu}{2 a} \sum_{j=1}^{N}\left(\phi_{j+1}-\phi_{j}\right)^{2}$ illustrates a fact that for a more elaborate discretization requires explicit calculations: the Laplacian does not uniquely determine the Lyapunov function [34-36].

Equation (22) has also been written in [14], although with different goals than ours. Their interest was to analyze the strong coupling limit via mapping to the directed polymer problem.

\section{An accurate consistent discretization}

Since the proposals of [60] already involve next-tonearest neighbors, one may seek for a prescription that minimizes the numerical error. An interesting choice for the Laplacian is [65]

$$
\frac{1}{12 a^{2}}\left[16\left(\phi_{j+1}+\phi_{j-1}\right)-\left(\phi_{j+2}+\phi_{j-2}\right)-30 \phi_{j}\right]
$$


PAPers in Physics, vol. 5, ART. 050010 (2013) / H. S. Wio et al.

which has the associated discrete form for the KPZ For the discretization scheme in Eq. (23), this is term

$$
\begin{aligned}
\left(\partial_{x} \phi\right)^{2}= & \frac{1}{24 a^{2}}\left\{1 6 \left[\left(\phi_{j+1}-\phi_{j}\right)^{2}\right.\right. \\
& \left.+\left(\phi_{j}-\phi_{j-1}\right)^{2}\right] \\
- & {\left.\left[\left(\phi_{j+2}-\phi_{j}\right)^{2}+\left(\phi_{j}-\phi_{j-2}\right)^{2}\right]\right\} } \\
& +\mathcal{O}\left(a^{4}\right) .
\end{aligned}
$$

Replacing this into the first line of Eq. (24), we obtain Eq. (25). Since this discretization scheme fulfills the consistency conditions, it is accurate up to $\mathcal{O}\left(a^{4}\right)$ corrections, and its prescription is not more complex than other known proposals, we expect that it will be the convenient one to use when high accuracy is required in numerical schemes [34-36].

\section{Relation with the Lyapunov functional}

In Sect. III we have indicated the form of the NEP for KPZ, and the way in which the functionals $\mathcal{F}[\phi]$ and $\mathcal{F}[h]$ are related [46]. According to the previous results, we can write the discrete version of Eq. (8) as

$$
\begin{gathered}
\mathcal{F}[h]=\frac{\lambda^{2}}{8 \nu} \frac{1}{2 a} \sum_{j} e^{\frac{\lambda}{\nu} h_{j}}\left[\left(h_{j+1}-h_{j}\right)^{2}\right. \\
\left.+\left(h_{j}-h_{j-1}\right)^{2}\right] .
\end{gathered}
$$

Introducing this expression into $\partial_{t} h_{j}=\Gamma_{j} \frac{\delta \mathcal{F}[h]}{\delta h_{j}}$, and through a simple algebra, we obtain Eq. (23). This reinforces our previous result, and moreover indicates that the discrete variational formulation naturally leads to a consistent discretization of the KPZ equation.

\section{The fluctuation-dissipation relation}

This relation is, together with Galilean invariance, a fundamental symmetry of the one-dimensional KPZ equation. It is clear that both symmetries are recovered when the continuum limit is taken in any reasonable discretization scheme. Thus, an accurate enough partition must yield suitable results.

The stationary probability distribution for the $\mathrm{KPZ}$ problem in $1 \mathrm{D}$ is known to be $[6,7]$

$$
\mathcal{P}_{\text {stat }}[h] \sim \exp \left\{-\frac{\nu}{2 \gamma} \int d x\left(\partial_{x} h\right)^{2}\right\} .
$$

$\sim \exp \left\{\frac{\nu}{2 \varepsilon} \frac{1}{2 a} \sum_{j}\left[\left(h_{j+1}-h_{j}\right)^{2}+\left(h_{j}-h_{j-1}\right)^{2}\right]\right\}$.

Inserting this expression into the stationary Fokker-Planck equation, the only surviving term has the form

$$
\begin{aligned}
\frac{1}{2 a^{3}} \sum_{j} & {\left[\left(h_{j+1}-h_{j}\right)^{2}+\left(h_{j}-h_{j-1}\right)^{2}\right] } \\
& \times\left[h_{j+1}-2 h_{j}+h_{j-1}\right] .
\end{aligned}
$$

The continuum limit of this term is $\int d x\left(\partial_{x} h\right)^{2} \partial_{x}^{2} h$, that is identically zero $[6,7]$. A numerical analysis of Eq. (28) indicates that it is several orders of magnitude smaller than the value of the exponents' pdf [in Eq. (27)], and typically behaves as $\mathcal{O}(1 / N)$, where $N$ is the number of spatial points used in the discretization. Moreover, it shows an even faster approach to zero if expressions with higher accuracy [like Eqs. (25) and (26)] are used for the differential operators. In addition, when the discrete form of $\left(\partial_{x} h\right)^{2}$ from [60] is used together with its consistent form for the Laplacian, the fluctuationdissipation relation is not exactly fulfilled. This indicates that the problem with the fluctuationdissipation theorem in $1+1$, discussed in $[18,60]$ can be just circumvented by using more accurate expressions.

\section{Galilean invariance}

This invariance means that the transformation

$$
x \rightarrow x-\lambda v t, \quad h \rightarrow h+v x, \quad F \rightarrow F-\frac{\lambda}{2} v^{2},
$$

where $v$ is an arbitrary constant vector field, leaves the KPZ equation invariant. The equation obtained using the classical discretization

$$
\partial_{x} h \rightarrow \frac{1}{2 a}\left(h_{j+1}-h_{j-1}\right),
$$

is invariant under the discrete Galilean transformation

$$
j a \rightarrow j a-\lambda v t, \quad h_{j} \rightarrow h_{j}+v j a, \quad F \rightarrow F-\frac{\lambda}{2} v^{2} .
$$


PAPers in Physics, vol. 5, ART. 050010 (2013) / H. S. Wio et al.

However, the associated equation is known to be numerically unstable [14], at least when $a$ is not small enough. Besides, Eq. (23) is not invariant under the discrete Galilean transformation. In fact, the transformation $h \rightarrow h+v j a$ yields an excess term which is compatible with the gradient discretization in Eq. (30); however, this discretization does not allow to recover the quadratic term in Eq. (23), indicating that this finite-difference scheme is not Galilean-invariant.

Since Eq. (21) is invariant under the transformation indicated in Eq. (31), it is the nonlinear Hopf-Cole transformation (within the present discrete context) which is responsible for the loss of Galilean invariance. Note that these results are independent of whether we consider this discretization scheme or a more accurate one.

Galilean invariance has always been associated with the exactness of the one-dimensional KPZ exponents, and with a relation that connects the critical exponents in higher dimensions [68]. If the numerical solution obtained from a finite-difference scheme as Eq. (23), which is not Galilean invariant, yields the well known critical exponents, this will be an indicative that Galilean invariance is not strictly necessary to get the KPZ universality class. The numerical results presented in [34-36] clearly show that this is the case.

We will not discuss here the simulation procedure but only indicate that to make the simulations we introduced a discrete representation of $h(x, t)$ along the substrate direction $x$ with lattice spacing $a=1$, and that a standard second-order Runge-Kutta algorithm (with periodic boundary conditions) was employed (see [66]). In [34-36] it was shown that all the cases (consistent or not) exhibit the same critical exponents. Moreover, we want to note that the discretization used in Refs. [60], which also violates Galilean invariance, yields the same critical exponents too. Additionally, stochastic differential equations which are not explicitly Galilean invariant have been shown to obey the relation $\alpha+z=2$ ([33], see also next section). Hence, our numerical analysis indicates that there are discrete schemes of the KPZ equation which, even not obeying Galilean invariance, show KPZ scaling.

The moral from the present analysis is clear: due to the locality of the Hopf-Cole transformation, the discrete forms of the Laplacian and the nonlinear (KPZ) term cannot be chosen independently; more- over, the prescriptions should be the same, regardless of the fields they are applied to. For further details we refer to [34-36].

\section{Renormalization-group analysis for Fluctuations}

In section III we have built a gradient flow counterpart of the deterministic KPZ equation. In this section we consider the corresponding stochastically forced gradient flow

$$
\partial_{t} u=-\frac{\delta \mathcal{I}}{\delta u}+\xi(x, t)
$$

with the density indicated in Eq. (16). We obtain the KPZW equation, which is the following SPDE

$$
\partial_{t} u=\nu \nabla^{2} u-\frac{\lambda}{6}(\nabla u)^{2}-\frac{\lambda}{3} u \nabla^{2} u+\xi(x, t) .
$$

Our present goal will be to analyze the scaling behavior of the fluctuations of the solution to this equation.

Since Eq. (33) is nonlinear, we focus on a perturbative technique. We choose the dynamic renormalization group as employed in [67,68]. Employing this method, we find at one-loop order the following flow equations [47]

$$
\begin{aligned}
& \frac{d \lambda}{d \ell}=\lambda(\alpha+z-2) \\
& \frac{d \nu}{d \ell}=\nu\left(z-2-\frac{1}{36} \frac{\lambda^{2} D}{\nu^{3}} K_{d} \frac{1-d}{d}\right) \\
& \frac{d \gamma}{d \ell}=\gamma\left(z-d-2 \alpha+\frac{K_{d}}{72} \frac{\lambda^{2} \gamma}{\nu^{3}}\right)
\end{aligned}
$$

where $K_{d}=S_{d} /(2 \pi)^{d}, S_{d}=2 \pi^{d / 2} / \Gamma(n / 2)$ is the surface area of the $d$-dimensional unit sphere, and $\Gamma$ is the gamma function. We find that the coupling constant $\bar{g}:=K_{d} \lambda^{2} \gamma / \nu^{3}$ obeys the one-loop differential equation

$$
\frac{d \bar{g}}{d \ell}=(2-d) \bar{g}+\frac{6-5 d}{72 d} \bar{g}^{2},
$$

revealing that the critical dimension of this model is $d_{c}=2$ as could be anticipated by means of power counting. For $d>2$ the coupling constant approaches zero exponentially fast in the scale $\ell$; for $d=2$, this approach is algebraic. So for these dimensions one expects the large-scale space-time 
PAPers in Physics, vol. 5, ART. 050010 (2013) / H. S. Wio et al.

properties of Eq. (33) to be dominated by its linear counterpart (up to marginal corrections in $d=2$ ). In $d=1$, the coupling constant runs to infinity for finite $\ell$, suggesting the presence of a nonperturbative fixed point (as the one in the KPZ equation for $d=2$ ).

The values of the critical exponents which yield scale invariance can be formally calculated by identifying with zero the right hand sides of Eqs. (34)(36). We get

$$
\begin{aligned}
\alpha & =\frac{2(2-d)(1-d)}{6-5 d} \\
z & =\frac{12-10 d-2(2-d)(1-d)}{6-5 d}
\end{aligned}
$$

which in particular obey the relation $\alpha+z=2$ in any dimensionality, despite the fact that Eq. (33) does not obey any sort of Galilean invariance. We note that in both $d=1$ and $d=2$ it is $\alpha=0$ and $z=2$, whereas $\alpha$ becomes negative in higher dimensions. Hence, in all dimensions, the exponent $\alpha$ indicates that the interface is either flat or at most marginally rough. The values for $d=1$ make both diffusion and nonlinearity in Eq. (33) invariant under the scale transformation $\{x, t, u\} \rightarrow$ $\left\{b x, b^{z} t, b^{\alpha} u\right\}$, as far as $b>1$. In this case, the noise grows with the scale (a fact that might explain the growth of the coupling constant in the renormalization group flow). In $d=2$, the exponents are those of the linear equation. An interesting result is that for $d=0$, the exponents become those of the KPZ equation: $\alpha=2 / 3$ and $z=4 / 3$, although this limit is highly singular for Eq. (37). Of course, these results have been obtained by means of a perturbative dynamic renormalization group and could be modified by non-perturbative contributions. One possible path to study such a possibility could be to adapt some non perturbative renormalization group techniques used for KPZ [69] to the present KPZW case.

Among all the results in this section, we would like to highlight the one given by Eq. (34). We recall that the $\mathrm{RG}$ analysis of the KPZ equation yields non-renormalization of the vertex and renormalization of propagator and noise. Our variational equation yields exactly the same result. Vertex non-renormalization at one-loop order is expressed by Eq. (34). The origin of this result is analogous to that of its equivalent in the KPZ equation: three non-vanishing Feynman diagrams con- tribute to vertex renormalization, but they cancel out each other [5] (a fact that has been traditionally attributed to the Galilean invariance of the KPZ equation). Here we have shown that the same result appears in a SPDE that is not even invariant under the translation $u \rightarrow u+$ constant.

\section{Stability}

We have carried out the NEP expansion about a constant solution of the KPZ equation and found that constants are still solutions to KPZW (Eq. (17)). In this section we will study the linear stability of such solutions. We start considering the solution

$$
u(x, t)=c+\epsilon v(x, t)
$$

where $c$ is an arbitrary constant and $\epsilon$ is the small parameter. Substituting in Eq. (17), we find

$$
\partial_{t} v=\frac{3 \nu-\lambda c}{3} \nabla^{2} v
$$

at first order in $\epsilon$. So $v$ obeys a diffusion equation whose diffusion constant depends on $c$. For $c<3 \nu / 2 \lambda$, the diffusion constant is positive and correspondingly the constant solution is linearly stable. For $c>3 \nu / 2 \lambda$, the diffusion constant is negative and consequently the constant solution is unstable. Furthermore, in this case the problem becomes linearly ill posed.

Since for large values of $c$, the problem becomes linearly ill posed, numerical solutions are not available. In order to solve this disadvantage, we could include a higher order term in our problem. We concentrate on the gradient flow

$$
\partial_{t} u=-\frac{\delta \mathcal{J}}{\delta u}+\xi(x, t)
$$

with density

$$
\begin{gathered}
\mathcal{J}[u]=\frac{\nu}{2} \int \mathrm{d} x(\nabla u)^{2}-\frac{\lambda}{6} \int \mathrm{d} x u(\nabla u)^{2} \\
+\frac{\mu}{2} \int \mathrm{d} x\left(\nabla^{2} u\right)^{2},
\end{gathered}
$$

leading to the following equation

$\partial_{t} u=\nu \nabla^{2} u-\frac{\lambda}{6}(\nabla u)^{2}-\frac{\lambda}{3} u \nabla^{2} u-\mu \nabla^{4} u+\xi(x, t)$. 
Note that the deterministic counterpart of this fourth-order equation can be considered as a variational version of the Kuramoto-Sivashinsky equation. It is worth remarking that this ad hoc construction resembles the one that, as indicated in [46], could more formally be obtained by considering the expansion of a nonlocal, short range interaction.

The regime of linear stability/instability of this equation is identical to that of Eq. (33) but in this case, the problem is always linearly well posed. Furthermore, the term proportional to $\mu$ is presumably irrelevant in the large spatiotemporal scale (as simple power counting of the linear terms reveals) so the results of the previous RG analysis could possibly hold for this case too. Anyway, due to the presence of the deterministic instability, further analysis are needed in order to assure this (note that both linear terms in the equation are stabilizing and that this instability has its origin in the vertex structure).

\section{Crossover: a path integral point of view}

Another recently discussed related aspect [70] is based in a path-integral Monte Carlo-like method for the numerical evaluation of the mean rugosity and other typical averages whose approach, which radically differs from one introduced before [71], exploits some of our previous results [34-36]. Here we limit ourselves to quote the temporally $(\mu)$ and spatially $(j)$ discrete form of the "stochastic action"

$$
\begin{aligned}
\mathbf{S}[h]= & \frac{1}{2 \tau} \sum_{j, \mu}\left\{h_{j, \mu+1}-h_{j, \mu}\right. \\
& \left.-\tau\left[\alpha \mathbf{L}_{j, \mu+1}+(1-\alpha) \mathbf{L}_{j, \mu}\right]\right\}^{2} \\
& -2 \nu \alpha N t \\
& -\tau \alpha \frac{\lambda}{2} \sum_{j, \mu}\left[h_{j+1, \mu}-2 h_{j, \mu}+h_{j-1, \mu}\right],
\end{aligned}
$$

and briefly discuss the obtained numerical results. $\tau$ is the time step, $0<\alpha<1$ a time-discretization parameter meant to be fixed for explicit calculation

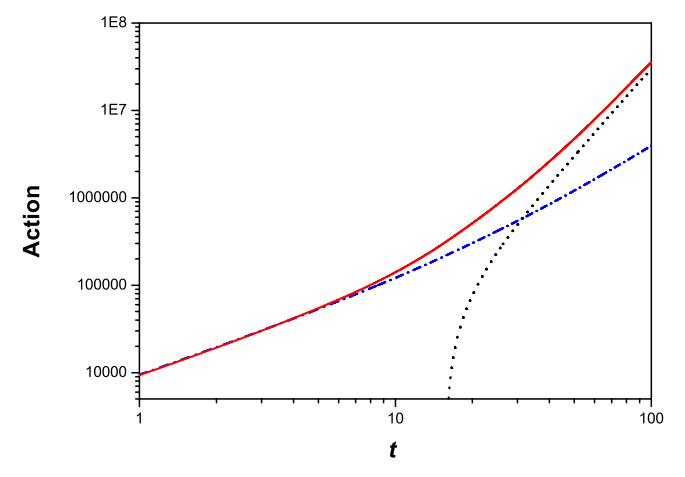

Figure 1: Crossover-like behavior from EW to KPZ regime for $\lambda=1$, on a lattice of 1028 sites $(\nu=D=$ 1). Red solid line: KPZ action; blue dash-dotted line: EW action; black-dotted line: difference.

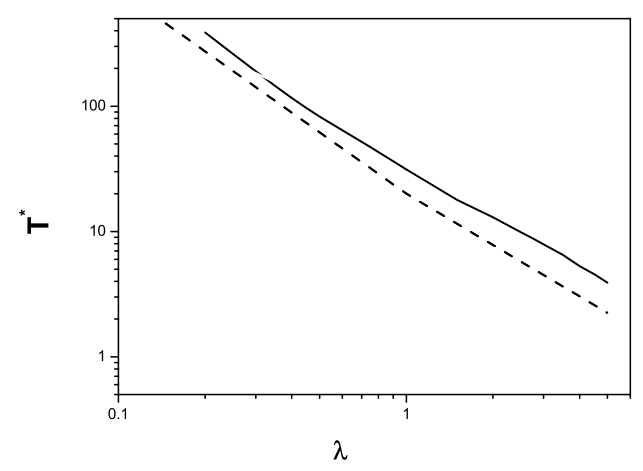

Figure 2: Same data as the previous figure. Solid line: time $T^{*}$ vs. $\lambda$; dashed line: trend for $T^{*} \sim$ $\lambda^{-1.35}$, included for comparison.

$$
\begin{aligned}
& {[72,73] \text {, and } \mathbf{L}_{j, \mu} \text { the "stochastic Lagrangian" }} \\
& \qquad \begin{aligned}
\mathbf{L}_{j, \mu}= & \nu\left(h_{j+1, \mu}-2 h_{j, \mu}+h_{j-1, \mu}\right) \\
& +\frac{\lambda}{4}\left[\left(h_{j+1, \mu}-h_{j, \mu}\right)^{2}\right. \\
& \left.+\left(h_{j, \mu}-h_{j-1, \mu}\right)^{2}\right]
\end{aligned}
\end{aligned}
$$

Figure 1 shows the crossover-like behavior from the Edwards-Wilkinson (EW) regime to the KPZ one. We take as estimator of such a transition the time at which the difference (dotted black line) between KPZ (red solid curve) and EW actions (blue 
PAPers in Physics, vol. 5, ART. 050010 (2013) / H. S. Wio et al.

dash-dotted line) crosses the EW one (it grossly coincides with the time at which the asymptotes cross). This estimator numerically agrees neither with the results in [10] (where a value of $\phi \sim 4$ was found) nor with the one in [24] (with $\phi \sim 3$, but corresponding to a $2 \mathrm{D}$ case). In Fig. 2 we have plotted the dependence of this estimator on $\lambda$. For comparison, we have also included the trend for $\lambda^{-\phi}$ with $\phi=1.35$ (dotted line). Preliminary results for $\lambda>7$ seem to indicate a marked change in the value of $\phi$, maybe a hint that the system is entering a strong coupling region [71].

\section{Short-time propagator}

Our aim here is to work out a variant of the method introduced in [70] by exploiting the first form of Lyapunov functional found in [46], namely Eq. (10), that leads us to Eq. (11), the full KPZ equation.

Whereas Eq. (45) is valid whatever the value of $\tau$, we now seek for a simpler expression valid for $\tau \ll 1$. This idea parallels in some sense other studies in the literature [71], but here we exploit the functional $\mathcal{F}[h]$ of Eq. (8). We denote as $\{h\}=\left(h_{1, \mu}, h_{2, \mu}, \ldots, h_{j, \mu}, \ldots, h_{N, \mu}\right)$ the interface configuration at time $\mu$. The transition pdf between patterns $h_{0}$ at $t_{0}$ and $h_{f}$ at $t_{f}$ can be written as

$$
\begin{aligned}
& P\left(\left\{h_{f}\right\}, t_{f} \mid\left\{h_{0}\right\}, t_{0}\right)= \\
& \quad \int \mathcal{D}[h] \exp \left(-\frac{1}{\gamma} \int_{t_{0}}^{t_{f}} \mathcal{L}[h, \dot{h}]\right),
\end{aligned}
$$

with

$$
\begin{aligned}
\mathcal{L}[h, \dot{h}]=\frac{1}{2} & \int_{0}^{L} d x\left[\left(\partial_{t} h+\Gamma[h] \frac{\delta}{\delta h} \mathcal{F}[h]\right)^{2}\right. \\
& \left.+\alpha \frac{\delta}{\delta h}\left(\Gamma[h] \frac{\delta}{\delta h} \mathcal{F}[h]\right)\right]
\end{aligned}
$$

whose discrete form is given by Eq. (46). A key observation is the (temporally and spatially) "diagonal" character of Eq. (9), highlighted in its discrete version

$$
\dot{h}_{j}(t)=-\Gamma_{j} \frac{\delta \mathcal{F}}{\delta h_{j}}+\sqrt{\gamma} \xi_{j}(t) .
$$

Guided by Eq. (46), we propose the following form of $P\left(\left\{h_{f}\right\}, t_{f} \mid\left\{h_{0}\right\}, t_{0}\right)$ for $\tau \ll 1$, or short-time propagator (STP)

$$
\begin{aligned}
& P\left(h_{f}, \tau \mid h_{0}, 0\right)= \\
& \int_{h_{0}}^{h_{f}} \mathcal{D}[h] e^{\left[-\frac{1}{2 \gamma} \int_{0}^{\tau} d s \int_{0}^{L} d x\left(\partial_{t} h+\Gamma \frac{\delta \mathcal{F}}{\delta h}\right)^{2}\right]} \\
& \approx \exp \left\{-\frac{\tau}{2 \gamma} \int_{0}^{L} d x\right. \\
& \left.\quad\left[\left(\frac{h_{f}-h_{0}}{\tau}+\frac{1}{2}\left[\Gamma_{f} \frac{\delta \mathcal{F}}{\delta h_{f}}+\Gamma_{0} \frac{\delta \mathcal{F}}{\delta h_{0}}\right]\right)^{2}\right]\right\} .
\end{aligned}
$$

Here, for simplicity, we have chosen a discretization with $\alpha=0$. As it is well known [72,73], the Jacobian of the transformation from the noise variable to the height variable depends on $\alpha$. With this choice, the Jacobian results equal to 1.

Incidentally, the form in Eq. (50) coincides with the discretization used in [71] for determining the least-action trajectory. The "quasi-Gaussian" character of this STP is better evidenced in the following approximate form

$$
\begin{gathered}
P\left(h_{f}, t_{f}=\tau \mid h_{0}, t_{0}=0\right) \sim e^{\left[-\frac{1}{2 \gamma \tau} \int_{0}^{L} d x\left(h_{f}-h_{0}\right)^{2}\right]} \\
\times\left\{1-\frac{1}{2 \gamma} \int_{0}^{L} d x\left[( h _ { f } - h _ { 0 } ) \frac { 1 } { 2 } \left(\Gamma_{f} \frac{\delta \mathcal{F}}{\delta h_{f}}\right.\right.\right. \\
\left.\left.\left.\quad+\Gamma_{0} \frac{\delta \mathcal{F}}{\delta h_{0}}\right)+\mathcal{O}(\tau)\right]\right\}
\end{gathered}
$$

where the exponential term has been separated out since it is of order $\tau^{-1}$, whereas the following two are of order $\tau^{0}$ and $\tau^{1}$, respectively (of lesser weight and negligible respectively, in the limit $\tau \rightarrow 0$ ). It is worth remarking that the term that could come from the Jacobian is also of order $\tau^{1}$.

It is easy to check that we can recover the known FPE from the proposed form of STP (adopting $\alpha=0$ for simplicity). We will not reiterate this calculation here. An immediate result of this form is that at very short times, behavior of the EdwardsWilkinson type is obtained

$$
\sqrt{\left\langle h^{2}\right\rangle} \approx \tau^{\frac{1}{2}} .
$$

\section{Conclusions}

Herein, in addition to reviewing some recent results $[34-36,47,70]$, we have furthered the study in [46], where it was shown that the deterministic KPZ 
PAPers in Physics, vol. 5, ART. 050010 (2013) / H. S. Wio et al.

equation admits a Lyapunov functional, and a (formal) definition of a nonequilibrium potential was introduced. We have carried out a Taylor expansion of such a nonequilibrium potential, what led us to a different equation of motion than the KPZ one, the KPZW which is an exact gradient flow and has an explicit density. In particular, it has a lower degree of symmetry: it is neither Galilean invariant, nor even translational invariant. The critical exponents determining its scaling properties were obtained through a one-loop dynamic renormalization group analysis. These exponents fulfill the same scaling relation as the KPZ equation, $\alpha+z=2$, traditionally attributed to the Galilean invariance of the latter. The fact that the same scaling relation arises in a SPDE (i.e., the KPZW) that is not only non-Galilean invariant but even non-invariant under the translation $u \rightarrow u+$ constant supports recent theoretical and numerical results indicating that Galilean invariance does not necessarily play the relevant role previously assumed in defining the universality class of the KPZ equation and different nonequilibrium models [30-36].

We have, moreover, analyzed the stability properties of the solutions to the present equation, finding the threshold condition for the appearance of diffusive instabilities, which indicates that in this case the problem becomes linearly ill posed. After considering the simplest way to correct such an illposed problem, we have met a kind of KuramotoSivashinsky equation, resembling the one that, as indicated in [46], could be obtained by considering a nonlocal, short range interaction. This equation has an exact gradient flow structure with an explicit density. Furthermore, when subject to stochastic forcing, its scaling properties could be formally described by the same critical exponents because the stabilizing term is irrelevant in the large scale from a dimensional analysis viewpoint.

Exploiting some elements of a path integral description of the problem, we have also shown what seems to be a simple form of viewing and studying the crossover from the EW to the KPZ regimes.

The present review-like study aims to open new points of view on, as well as alternative routes to study, the KPZ problem. Among the many aspects to be further studied, an interesting one is to test the (kind) of stability of the recently found exact solutions [20-23] by exploiting the indicated form of the NEP.
Acknowledgements - Financial support from MINECO (Spain) is especially acknowledged, through Projects PRI-AIBAR-2011-1323 (which enabled international cooperation), FIS2010-18023 (HSW and CE) and RYC-2011-09025 (CE). Also acknowledged is the support from CONICET, UNC (JAR) and UNMdP (RRD) of Argentina. Collaboration with M.S. de la Lama and E. Korutcheva during different stages of this research is highly appreciated.

[1] L D Landau, E M Lifshitz, Statistical physics, Butterworth Heinemann, Oxford, 1980.

[2] L P Kadanoff, W Götze, D Hamblen, R Hecht, E A S Leis, V V Palciauskas, M Rayl, J Swift, D Aspnes, J Kane, Static phenomena near critical points: Theory and experiment, Rev. Mod. Phys. 39, 395 (1967).

[3] K G Wilson, J Kogut, The renormalization group and the $\epsilon$ expansion, Phys. Rep. 12, 75 (1974); K G Wilson, The renormalization group: Critical phenomena and the Kondo problem, Rev. Mod. Phys. 47, 773 (1975).

[4] J Marro, R Dickman, Nonequilibrium phase transitions in lattice models, Cambridge U. Press, Cambridge, UK (1999); M Henkel, H Hinrichsen, S Lübeck, Nonequilibrium phase transitions - I, Springer, Berlin (2008); M Henkel, M Pleimling, Nonequilibrium phase transitions - II, Springer, Berlin (2010); G Ódor, Universality in nonequilibrium lattice systems, World Scientific, Singapore (2008).

[5] M Kardar, G Parisi, Y-C Zhang, Dynamic scaling of growing interfaces, Phys. Rev. Lett. 56, 889 (1986).

[6] T Halpin-Healy, Y-C Zhang, Kinetic roughening phenomena, stochastic growth, directed polymers and all that. Aspects of multidisciplinary statistical mechanics, Phys. Rep. 254, 215 (1995).

[7] A-L Barabási, H E Stanley, Fractal concepts in surface growth, Cambridge U. Press, Cambridge, UK (1995).

[8] V Gurarie, A Migdal, Instantons in the Burgers equation, Phys. Rev. E 54, 4908 (1996). 
PAPers in Physics, vol. 5, ART. 050010 (2013) / H. S. Wio et al.

[9] M Kardar, Replica Bethe ansatz studies of twodimensional interfaces with quenched random impurities, Nucl. Phys. B 290, 582 (1987).

[10] B M Forrest, R Toral, Crossover and finitesize effects in the (1+1)-dimensional KardarParisi-Zhang equation, J. Stat. Phys. 70, 703 (1993).

[11] M Beccaria, G Curci, Numerical simulation of the Kardar-Parisi-Zhang equation, Phys. Rev. E 50, 4560 (1994).

[12] K Moser, D E Wolf, Vectorized and parallel simulations of the Kardar-Parisi-Zhang equation in $3+1$ dimensions, J. Phys. A 27, 4049 (1994).

[13] M Scalerandi, P P Delsanto, S Biancotto, Time evolution of growth phenomena in the KPZ model, Comput. Phys. Commun. 97, 195 (1996).

[14] T J Newman, A J Bray, Strong-coupling behaviour in discrete Kardar-Parisi-Zhang equations, J. Phys. A 29, 7917 (1996).

[15] C Appert, Universality of the growth velocity distribution in $1+1$ dimensional growth models, Comput. Phys. Commun. 121-122, 363 (1999).

[16] E Marinari, A Pagnani, G Parisi, Critical exponents of the $K P Z$ equation via multi-surface coding numerical simulations, J. Phys. A 33, 8181 (2000).

[17] T J Oliveira, S G Alves, S C Ferreira, KardarParisi-Zhang universality class in $(2+1) d i$ mensions: Universal geometry-dependent distributions and finite-time corrections, Phys. Rev. E 87, 040102 (2013).

[18] L Giada, A Giacometti, M Rossi, Pseudospectral method for the Kardar-ParisiZhang equation, Phys. Rev. E 65, 036134 (2002).

[19] V G Miranda, F D A Aarão Reis, Numerical study of the Kardar-Parisi-Zhang equation, Phys. Rev. E 77, 031134 (2008).
[20] T Sasamoto, H Spohn, One-dimensional Kardar-Parisi-Zhang equation: an exact solution and its universality, Phys. Rev. Lett. 104, 230602 (2010).

[21] T Sasamoto, H Spohn, The 1+1-dimensional Kardar-Parisi-Zhang equation and its universality class, J. Stat. Mech. P11013 (2010).

[22] G Amir, I Corwin, J Quastel, Probability distribution of the free energy of the continuum directed random polymer in $1+1$ dimensions, Commun. Pure Appl. Math. 64, 466 (2011).

[23] P Calabrese, P Le Doussal, Exact solution for the Kardar-Parisi-Zhang equation with flat initial conditions, Phys. Rev. Lett. 106, 250603 (2011).

[24] H Guo, B Grossmann, M Grant, Crossover scaling in the dynamics of driven systems, Phys. Rev. A 41, 7082 (1990).

[25] C M Horowitz, E V Albano, Relationships between a microscopic parameter and the stochastic equations for interface's evolution of two growth models, Eur. Phys. J. B 31, 563 (2003).

[26] F D A Aarão Reis, Scaling in the crossover from random to correlated growth, Phys. Rev. E 73, 021605 (2006).

[27] S Bustingorry, L Cugliandolo, J L Iguain, Out-of-equilibrium relaxation of the EdwardsWilkinson elastic line, J. Stat. Mech. P09008 (2007); S Bustingorry, Aging dynamics of nonlinear elastic interfaces: The Kardar-ParisiZhang equation, J. Stat. Mech. 10002 (2007).

[28] S Bustingorry, P LeDoussal, A Rosso, Universal high-temperature regime of pinned elastic objects, Phys. Rev. B 82, 140201 (2010); S Bustingorry, A B Kolton, T Giamarchi, Random-manifold to random-periodic depinning of an elastic interface, Phys. Rev. B 82, 094202 (2010).

[29] M Henkel, J D Noh, N Pleimling, Phenomenology of aging in the Kardar-Parisi-Zhang equation, Phys. Rev. E 85, 030102 (2012). 
PAPers in Physics, vol. 5, ART. 050010 (2013) / H. S. Wio et al.

[30] W D McComb, Galilean invariance and vertex renormalization in turbulence theory, Phys. Rev. E 71, 037301 (2005).

[31] A Berera, D Hochberg, Gauge symmetry and Slavnov-Taylor identities for randomly stirred fluids, Phys. Rev. Lett. 99, 254501 (2007).

[32] A Berera, D Hochberg, Gauge fixing, BRS invariance and Ward identities for randomly stirred flows, Nucl. Phys. B 814, 522 (2009).

[33] M Nicoli, R Cuerno, M Castro, Unstable nonlocal interface dynamics, Phys. Rev. Lett. 102, 256102 (2009).

[34] H S Wio, J A Revelli, $\mathrm{R}$ R Deza, C Escudero, M S de La Lama, KPZ equation: Galilean-invariance violation, consistency, and fluctuation-dissipation issues in real-space discretization, Europhys. Lett. 89, 40008 (2010).

[35] H S Wio, J A Revelli, R R Deza, C Escudero, M S de La Lama, Discretization-related issues in the Kardar-Parisi-Zhang equation: Consistency, Galilean-invariance violation, and fluctuation-dissipation relation, Phys. Rev. E 81, 066706 (2010).

[36] H S Wio, C Escudero, J A Revelli, R R Deza, M S de La Lama, Recent developments on the Kardar-Parisi-Zhang surface-growth equation, Phil. Trans. R. Soc. A 369, 396 (2011).

[37] T Sun, H Guo, M Grant, Dynamics of driven interfaces with a conservation law, Phys. Rev. A 40, 6763 (1989).

[38] J Villain, Continuum models of crystal growth from atomic beams with and without desorption, J. Phys. I (France) 1, 19 (1991).

[39] Z-W Lai, S Das Sarma, Kinetic growth with surface relaxation: Continuum versus atomistic models, Phys. Rev. Lett. 66, 2348 (1991).

[40] C Escudero, Geometric principles of surface growth, Phys. Rev. Lett. 101, 196102 (2008).

[41] C Escudero, E Korutcheva, Origins of scaling relations in nonequilibrium growth, J. Phys. A: Math. Theor. 45, 125005 (2012).
[42] R Graham, Weak noise limit and nonequilibrium potentials of dissipative dynamical systems, In: Instabilities and nonequilibrium structures, Eds. E Tirapegui, D Villaroel, D, Reidel Pub. Co., Dordrecht (1987).

[43] H S Wio, Nonequilibrium potential in reactiondiffusion systems, In: 4th Granada seminar in computational physics, Eds. P Garrido, J Marro, Pag. 135, Springer-Verlag, Berlin (1997).

[44] H S Wio, R R Deza, J M López, Introduction to stochastic processes and nonequilibrium statistical physics, Revised Edition, World Scientific, Singapore (2013).

[45] P Ao, Potential in stochastic differential equations: novel construction, J. Phys. A 37, L25 (2004).

[46] H S Wio, Variational formulation for the KPZ and related kinetic equations, Int. J. Bif. Chaos 19, 2813 (2009).

[47] C Escudero, E Korutcheva, H S Wio, R R Deza, J A Revelli, KPZ equation as a gradient flow: Nonequilibrium-potential expansion and renormalization-group treatment of fluctuations, unpublished.

[48] P Hohenberg, B Halperin, Theory of dynamic critical phenomena, Rev. Mod. Phys. 49, 435 (1977).

[49] G Grinstein, S K Ma, Surface tension, roughening, and lower critical dimension in the random-field Ising model, Phys. Rev. B 28, 2588 (1983).

[50] J Koplik, H Levine, Interface moving through a random background, Phys. Rev. B 32, 280 (1985).

[51] R Bruinsma, G Aeppli, Interface motion and nonequilibrium properties of the random-field Ising model, Phys. Rev. Lett. 52, 1547 (1984).

[52] D Kessler, H Levine, Y Tu, Interface fluctuations in random media, Phys. Rev. A 43, 4551 (1991). 
PAPers in Physics, vol. 5, ART. 050010 (2013) / H. S. Wio et al.

[53] H S Wio, S Bouzat, B Von Haeften, Stochastic resonance in spatially extended systems: the role of far from equilibrium potentials, Physica A 306, 140 (2002).

[54] H S Wio, R R Deza, Aspects of stochastic resonance in reaction-diffusion systems: The nonequilibrium-potential approach, Eur. Phys. J.-Spec. Top. 146, 111 (2007).

[55] H G E Hentschel, Shift invariance and surface growth, J. Phys. A: Math. Gen. 27, 2269 (1994).

[56] S J Linz, M Raible, P Hänggi, Stochastic field equation for amorphous surface growth, In: Stochastic processes in physics, chemistry, and biology, Eds. J A Freund, T Pöschel, 557, Pag. 473, Springer, Berlin (2000).

[57] J M López, M Castro, R Gallego, Scaling of local slopes, conservation laws, and anomalous roughening in surface growth, Phys. Rev. Lett. 94, 166103 (2005).

[58] M Castro, J Muñoz-García, R Cuerno, M M García-Hernández, L Vázquez, Generic equations for pattern formation in evolving interfaces, New J. Phys. 9, 102 (2007).

[59] E Hernández-García, T Ala-Nissila, M Grant, Interface roughening with a time-varying external driving force, Europhys. Lett. 21, 401 (1993).

[60] C-H Lam, F G Shin, Improved discretization of the Kardar-Parisi-Zhang equation, Phys. Rev. E 58, 5592 (1998); C-H Lam, F G Shin, Formation and dynamics of modules in a dual-tasking multilayer feed-forward neural network, Phys. Rev. E, 57, 6506 (1998).

[61] R Gallego, M Castro, J M López, Pseudospectral versus finite-difference schemes in the numerical integration of stochastic models of surface growth, Phys. Rev. E 76, 051121 (2007).

[62] S M A Tabei, A Bahraminasab, A A Masoudi, S S Mousavi, M R R Tabar, Intermittency of height fluctuations in stationary state of the Kardar-Parisi-Zhang equation with infinitesimal surface tension in $1+1$ dimensions, Phys. Rev. E 70, 031101 (2004).
[63] K Ma, J Jiang, C B Yang, Scaling behavior of roughness in the two-dimensional KardarParisi-Zhang growth, Physica A 378, 194 (2007).

[64] M S de la Lama, J M López, J J Ramasco, M A Rodríguez, Activity statistics of a forced elastic string in a disordered medium, J. Stat. Mech., P07009 (2009).

[65] M Abramowitz, I A Stegun, Handbook of mathematical functions: With formulas, graphs, and mathematical tables, Pag. 884, Dover, New Tork (1965).

[66] M San Miguel, R Toral, Stochastic effects in physical systems, In: Instabilities and nonequilibrium structures VI, Eds. E Tirapegui, J Martínez-Mardones, R Tiemann, Pag. 35, Kluwer Academic Publishers (2000).

[67] D Forster, D R Nelson, M J Stephen, Largedistance and long-time properties of a randomly stirred fluid, Phys. Rev. A 16, 732 (1977).

[68] E Medina, T Hwa, $\mathrm{M}$ Kardar, Y-C Zhang, Burgers equation with correlated noise: Renormalization-group analysis and applications to directed polymers and interface growth, Phys. Rev. A 39, 3053 (1989).

[69] L Canet, H Chate, B Delamotte, General framework of the non-perturbative renormalization group for non-equilibrium steady states, J. Phys. A 44, 495001 (2011); L Canet, H Chate, B Delamotte, N Wschebor, Nonperturbative renormalization group for the Kardar-Parisi-Zhang equation: General framework and first applications, Phys. Rev. E 84, 061128 (2011); Th Kloss, L Canet, N Wschebor, Nonperturbative renormalization group for the stationary Kardar-Parisi-Zhang equation: Scaling functions and amplitude ratios in 1+1, 2+1, and 3+1 dimensions, Phys. Rev. E 86, 051124 (2012).

[70] H S Wio, R R Deza, J A Revelli, C Escudero, $A$ novel approach to the $K P Z$ dynamics, Acta Phys. Pol. B 44889 (2013).

[71] H C Fogedby, W Ren, Minimum action method for the Kardar-Parisi-Zhang equation, Phys. Rev. E 80, 041116 (2009). 
PAPers in Physics, vol. 5, ART. 050010 (2013) / H. S. Wio et al.

[72] F Langouche, D Roekaerts, E Tirapegui, Functional integration and semiclassical expansions, D. Reidel Pub. Co., Dordrecht (1982).
[73] H S Wio, Path integrals for stochastic processes: An introduction, World Scientific, Singapore (2013). 